\title{
ANTECEDENTES Y EVOLUCIÓN HISTÓRICA, SOBRE EL TRABAJO DESARROLLADO EN EL SEMINARIO PERMANENTE DE HISTORIA DE LA ENFERMERÍA EN ASTURIAS
}

\author{
Carmen Chamizo Vega
}

\section{RESUMEN}

En este trabajo la autora describe la evolución de el seminario permanente de historia de la enfermeria de Asturias (España).

Palabras clave: historia de la enfermeria

ANTECEDENTS AND HISTORICAL EVOLUTION, ABOUT THE DEVELOPED WORK OF THE PERMANENT SEMINARY OF NURSING HISTORY IN ASTURIAS

\section{SUMMARY}

In this work the author describes the evolution of the permanent seminary of nursing history of Asturias (Spain)

Keywords: Nursing history

El Seminario permanente sobre historia de la enfermería se fundó en Madrid en 1989, unido al Departamento de Enfermería Fundamental, cuya profesora principal es Francisca Hernández Martín.

Hacia esa misma fecha, se tomó contacto con el Centre for Historical in Nursing de la Universidad de Pensilvania, en Filadelphia (EE.UU.). Este centro está a cargo del proyecto "la historia de la enfermería en los últimos 200 años" del International Nursing Council para 1999.

Estimulados por la existencia de dicho proyecto, diversos profesionales de Enfrermería empe- zamos a trabajar en las diferentes provincias españolas sobre dicho tema. En Asturias se constituyó un grupo en 1995, para conocer la evolución de los cuidados enfermeros en dicha provincia, a través de fuentes documentales y archivísticas, en el período de tiempo desde el reconocimiento legislativo de la profesión (1857) y la inclusión en la Univesidad (1977).

El grupo de trabajo está formado por:

- Manuel Ballesteros

- Veneranda Magdaleno

-Yolanda Cotiello

-Fernando Nodar

- Carmen Chamizo

-Actualmente se incorporarán 4 enfermeras más.

Entre los trabajos realizados hasta la fecha, se encuentran:

—El poster didáctico sobre la evolución legislativa de la Enfermería española y presentación en la bienal de Enfermeras Investigadoras W.E.N.R. ) en Estocolmo en 1996 y Helsinki 1998 de dos posters con contenido de la línea de investigación.

-El cuento de "La Historia de Paulina". Cuento para niños explicando la Historia de la Enfermería.

- La escuela de Puericultura del Instituto de Puericultura de Gijón (Editada por el Ayuntamiento de Gijón). 
- Realización del IV Congreso Nacional de Historia de la Enfermería en 1999.

-Presentación del Poster sobre las investigaciones de $H^{a}{ }^{a}$ de la Enfermería Española, en el C.I.E. de Londres en 1999.

-Colaboración con distintos profesionales para el estudio de la Historia de la Enfermería iberoamericana (Coloquio iberoamericano sobre $\mathrm{H}^{\text {a }}$ de la Enfermería en Agosto del 2000).

-Puesta en el aire de la Página de Historia de la Enfermería Española.

- Realización de una lista de correo sobre Historia de la Enfermería ( próximamente).

\section{PRESENTACIÓN Y SALUTACIÓN}

Buenos días a todos. Me gustaría comenzar dándoles las gracias por su presencia hoy aquí, y en especial a Pepe Siles, que en representación de los organizadores de este evento, ha tenido la amabilidad de invitarme. También decir que este trabajo es una pequeña muestra de la investigación que desde hace 6 años, hemos llevado a cabo y en la que han participado muchos y variados profesionales constituyendo en 1997 la Asociación de Historia de la Enfermería. A todas y cada una de las personas que con su aportación y apoyo han hecho posible este momento: Gracias.

\section{INTRODUCCIÓN}

En una ocasión leí una frase de Francisco Ventosa que decía: " una profesión que no conoce su Historia, es algo tan anómalo como un hombre sin memoria, que no guarda el menor recuerdo de los hechos de la vida pasada ". Algo parecido nos ocurría al comenzar el estudio. Conocíamos la historia de Florence de Nigtingale, pero había un vacío entre ella y nuestro ejercicio actual. Sabíamos que había algo más cercano que la escuela de Rubio Galí, algo más provincial e incluso más local ; ¿ quien impartía los cuidados enfermeros en Asturias ? ¿ como se formaba el personal de enfer- mería en Asturias ? ¿disponiamos de suficientes textos históricos para poder definir y situar la enfermería en Asturias?... Todas estas preguntas y más, se agolpaban en nuestras primeras reuniones de trabajo .

Aunque los primeros datos, que sobre formación de enfermeras, hemos encontrado, datan de 1600 y corresponden a tres mujeres de "Buena reputación "que son enviadas al hospitalillo de Alcalá de Henares para formarse como matronas o " parteras" ( A.G.A.P.A. ), no los tendremos en cuenta por definir el período de estudio, el comprendido entre el reconocimiento legal como profesión y la incorporación a la Universidad; es decir desde 1857 a 1977.

El estudio es histórico - descriptivo y la metodología se ha basado en la recogida y organización de material archivístico y bibliográfico, tanto de archivos y bibliotecas nacionales como provinciales.

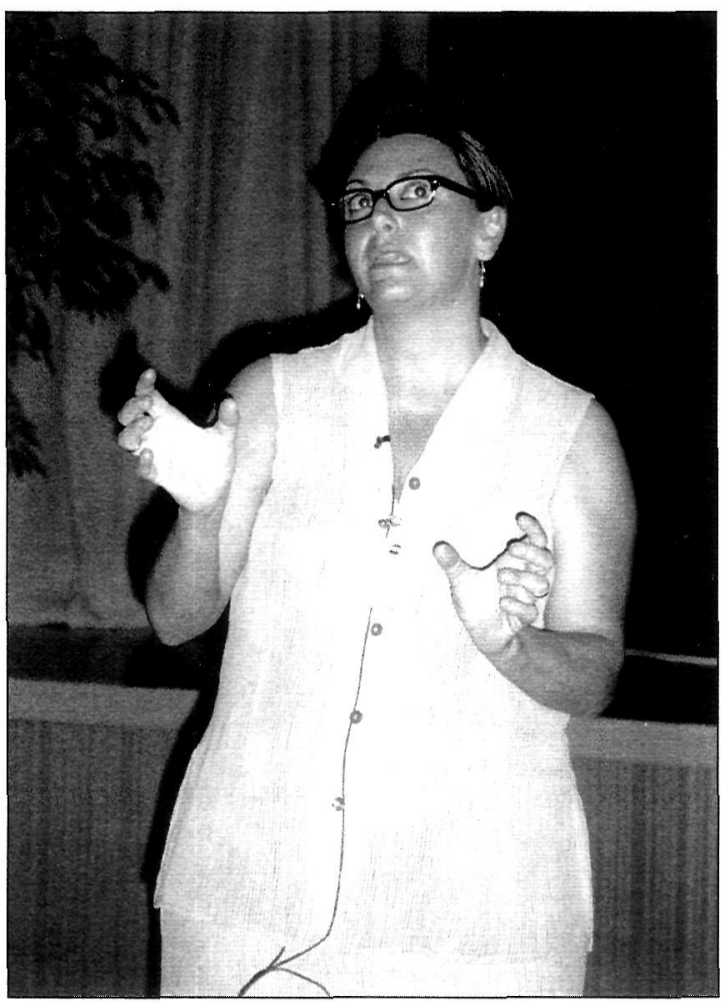


El aspecto más importante de este estudio es que ayuda a conocer la evolución académica de los cuidadores y los cuidados que dispensaron en Asturias, para asî identificar las influencias culturales y los valores, colocando las teorías dentro del contexto adecuado.

Por la extensión de los datos recogidos y para esta ocasion, he escogido por su interés y particularidad, la Escuela de Puericultura de la Gota de Leche de Gijón.

Finalmente les mostraré alguno de los trabajos novedosos y que hemos realizado para contribuir al conocimiento de la Historia de la Enfermería Española en diversos ámbitos nacionales e internacionales.

Así como ocurre con el microscopio o con la cámara de fotos, si no graduamos bien el objetivo, no sólo nos hace ver mal, sino que nos hace ver otra cosa, en la Historia acontece lo mismo ; cada época exige una acomodación peculiar de nuestro órgano intuitivo e intelectual y para ir " acomodándonos" . comenzaré con un repaso general a la evolución de las titulaciones de los profesionales de la Enfermería desde 1857 a 1977 en España, para " ir enfocando " y comprender mejor el interés de los datos provinciales que referiré posteriormente.

\section{EVOLUCIÓN DE LAS TITULACIONES DE LOS P. DE E.}

En tiempos de Carlos III, se retoma el control del sistema educativo, vertebrando la enseñanza en tres niveles. Apartir de aquí se suceden multitud de Planes (Calomarde, Duque de Rivas, Pidal, Mata ..) aprobándose los estudios de la Practica en el arte de Curar (R.D.10-10-1843) y Ministrantes (R.0.29-6-1846) ....hasta llegar a una disposición fundamental en la Historia de la Enfermeria : La Ley Moyano (1857), mediante la que se aprueban las carreras de Practicante y Matrona.
Tras la Restauración (1870) la Iglesia retoma el dominio de la educación y es posteriormente, Romanones quien mejora y crea las carreras medias e impulsa la unificación de la ensenanza de los Practicantes (1888 a 1904), prescribiéndose la obligatoriedad de aprobar un examen de ingreso para hacer la matricula del primer curso de Practicantes y Matronas y organizándose la enseñanza en dos años haciendo incapié en la necesidad de la seriedad en las prácticas hospialarias, para finalmente y ras los dos años superar un examen de reválida en las Facultades de Medicina. Se suceden una serie de disposiciones, que culmina con la aprobación de la Ley de Instrucción Pública en 1904 en la que se regulaban las profesiones sanitarias y donde se plasma legalmente, la Enfermria como profesión sanitaria , aunque en la práctica habitual la figura de la enfermera sólo empezó a ser considerada como profesional tras la unificación de las escuelas y los títulos a partir de 1915 con la R.O. del 7 de Mayo de 1915 .

En 1931 habian pasado más de 15 años desde la creación del título oficial de Enfermera, pero una orden ministerial en este año exige nuevamente, la unificación de los títulos de enfermera en todas las facultades de Medicina de la República, dándonos este hecho idea del retraso considerable del cumplimiento de la sistematización de la Enfermería.

Fué en los años de la II República cuado las enfermeras alcanzaron un estatus similar e incluso superior al de los practicantes y matronas, debiéndose este hecho, no sólo al proceso de formación de estos profesionales sino que hay que considerarlo en el marco global de la emancipación de la mujer y su inserción en los distintos sectores laborales. En este proceso estuvieron involucrados insignes institucionistas que ocuparon cargos de elevada responsabilidad en el Ministerio de Instrucción Pública. 
De lo expresado anteriormente se deduce que el proceso de regulación en los dos campos fundamentales de la Enfermería : el educativo y el laboral, se fué consolidadndo durante el primer tercio del s.XX, adquiriendo mayor importancia en los años veinte, pero la formación de los Profesionales de Enfermería, no alcanzó su cota más elevada hasta la llegada del período Republicano.

Tras la Guerra Civil, la situación de la Enfermería se caracterizó fundamentalmente por la vuelta a las actividades y valores tradicionales y un considerable menoscabo de los logros conseguidos durante la etapa republicana. Tanto los hombres, como las mujeres que estudiaban enfermería fueron formados bajo las consignas de la Falange, la Iglesia y el Estado, es decir que la trágica quiebra producida por el conflicto bélico significó una importante regresión, que afectó tanto a la sociedad española, en general, como al marco concreto de la Enfermería. Se retorna a la situación de actividad directamente relacionada con la división sexual del trabajo, recuperando su estatuto (discriminatorio) de profesión femenina, por imperativos ideológicos y sociales. Esta vuelta atrás, trajo consigo el incremento del número de religiosos y religiosas que recuperaron gran parte del protagonismo perdido dentro del campo competencial de la Enfermería.

Con la entrada en el Ministerio de Educación Nacional de Ruiz Giménez en 1951, se inicia el cambio hacia el modelo tecnocrático y aunque la confesionaidad de la enseñanza se reforzó mediante la firma del Concordato de 1953, existe cierta apertura de máxima trascendencia para la enfermería. Así se suceden diversas normativas que entre otras son la exigencia del título de bachiller elemental, unificación de los planes de estudio de enfermería y normativa de funcionamiento de las escuelas de A.T.S. En este contexto se produce una potenciación de las especialidades que no tuvo una adecuada vertebración laboral.
Conforme el país se iba abriendo al exterior y abandonando el sistema autárquico, se produjeron las primeras manifestaciones encontra del Régimen . Las revueltas estudiantiles de 1956 provocaron la salida de Ruiz Gimenez y en 1957, en la Ley sobre Ordenamiento de Enseñanzas Técnicas, se exponían los motivos sociales y económicos para justificar la necesidad de crear nuevas carreras de grado medio. En este contexto el modelo tecnocrático se afianzó gracias a que en los años sesenta hubo un cambio en la política sanitaria que propició la construcción de instalaciones sanitarias (triplicándose el número de centros sanitarios de 1963 a 1970). Este desarrollo tuvo consecuencias notables en la enseñanza de Enfermería, se hizo un gran esfuerzo frente al fuerte incremento de la demanda de personal de enfermería en tan poco tiempo y se impulsó el proceso de secularización del personal que trabajaba en los hospitales existentes. También en estos años se produjo, una evidente segregación de los profesionales de Enfermería en función del sexo, ejemplo de ello es que en los planes de estudio de los años sesenta y principio de los setenta se seguían contemplando asignaturas más estrictamente relacionadas con inmersión ideológica y dogmatización religiosa que con la enseñanza de la enfermería como eran : formación del espíritu nacional, religión y en las enfermeras materias relacionadas con las labores tradicionales de la mujer en el hogar. Si bien es cierto, que gracias al buen criterio de muchos profesores, estas materias eran habilmente esquivadas o se impartían de una manera marginal en algunas escuelas.

En 1968 toma posesión de su cargo en el Ministerio de Educación y Ciencia,Villar Palasí y en 1970 se promulgó la Ley General de Educación y Financiación de la Reforma Educativa, constituyendo esta Ley, el primer intento de dar una respuesta global a los planteamientos de la centenaria Ley Moyano. Se promueve la inclusión de los 


\section{$46 \cdot$ Cultura de los Cuidados}

estudios de primer ciclo en la Universidad. En este contexto, a la enfermería se le ofrece la posibilidad de elegir entre integrarse en los estudios de F.P. (de $2^{\circ}$ y $3^{\circ}$ grado) o adcribirse en la Universidad.

En Febrero de 1976 se constituyó la Comisión Interministerial encargada de la reforma de los estudios de A.T.S. integrándose en la misma miembros provinientes de diferentes organismos ( Dirección General de Sanidad, Ministerio de Educación y Ciencia, Instituto Nacional de Previsión, Sindicato de Actividades Sanitarias y las tres ramas del Consejo General de A.T.S. ), como miembros asesores estuvieron dos médicos y dos enfermeras, representantes de escuelas privadas y de la Comisión Nacional de Escuela de Enfermería, respectivamente. Para la elaboración del documento orientativo, los expertos utilizaron las directrices del Consejo de Europa del 27 de Junio de 1977, sobre coordinación de las disposiciones legales, reglamentarias y administrativas relativas a las actividades de los enfermeros/as de Cuidados Generales. Pero es la presión ejercida por el sector laboral y especialmente el hospitalario, la que logra que se constituya una Coordinadora Nacional que resultó determinante y posteriormente, mediante R. O. del 23 de Julio de 1977, se integran los estudios de Enfermería en la universidad.

\section{LA FORMACIÓN DE ENFERMERAS EN LA ESCUELA DE PUERICULTURA DE LA GOTA DE LECHE DE GIJÓN}

\section{-Reseña histórica:}

En Gijón, durante el siglo XIX se llevaron a cabo un gran número de mejoras sanitarias y de infraestructura que mejoró considerablemente la salud de todos los gijoneses reduciendo mucho su mortalidad; pero aún asi, en 1910, aunque la mortalidad de adultos estaba por debajo de la media nacional, la infantil era muy superior a la registrada en España con cifras de 290 por mil, mientras que la nacional era de 160 por mil nacidos vivos.
Así pues, tras crearse en 1904 por Real Orden, las Juntas de Protección de Menores, llamadas en su primera organización de Protección a la Infancia, se constituye la Junta de Gijón en Marzo de 1908 . Por la falta de ingresos su labor, inicialmente, queda reducida a vigilancia y tutela moral, poco eficiente y de acuerdo con las disponibilidades de la Beneficencia Municipal. Es a partir de 1914 y sobre todo a partir de 1917, cuando se concede a las Juntas el 5\% sobre espectáculos públicos, dando comienzo en las Juntas a una labor intensa de protección a la infancia. A primeros de 1922, es renovada la Junta, entrando a formar parte de ella, el vocal médico Avelino González, siendo nombrado secretario de la misma - Es cuando de modo definitivo, puede decirse que nació la Junta Local de Menores de Gijón, con ingresos propios a través de ideas como la creación de la Rifa Benéfica Pro-Infancia.

El Dr. Avelino González, nace en 1894, en Lamuño concejo de Cudillero y muere, en Gijón el 7 de Noviembre de 1978 . Estudia durante su infancia en Gijón y posteriormente cursa estudios de Medicina en Valladolid. Al finalizar, acude a Madrid donde se forma como puericultor en el Hospital del Dr. Suñer, que sería el fundador y primer Director de la Escuela Nacional de Puericultura y del que se consideraría discípulo y amigo. Alli conoce también al que sería jefe de la sección de Puericultura e higiene escolar de Sanidad Nacional, el Dr. Bosch Marín, benefactor de esta escuela y amigo de aquél. De la experiencia en Madrid viene a Gijón con ideas innovadoras, e incluso es allí donde la Reina Madre, María Cristina de Borbón, le hace la sugerencia de la creación de una Gota de Leche, donde se repartian gratuitamente botellines debidamente dosificados con leche pasteurizada y donde se atendian sanitariamente a las madres y a los niños hasta los 14 años.

En 1922 Don Avelino, propone la construcción de un edificio para Gota de Leche, la creación 
de una sala de maternidad y que se establezca en Gijón la cartilla higiénica de las madres. En 1923 pide la creación de la Casa - Cuna y en 1925, es propuesto por el alcalde de Gijón como director técnico del. Instituto de Puericultura de Gijón,realizándose ese mismo año su inaguración

Es en 1926, teniendo la Junta Local de protección de Menores, gran envergadura es cuando propone la creación de una Escuela Provincial de Puericultura, de un laboratorio y de una Biblioteca

Posteriormente, pide la creación de un comedor de embarazadas y madres que lactan, y la realización de un Parque Infantil Modelo y un Hogar Maternal e Infantil con escuela para madres, escuela maternal y refugio infantil.

\section{LA ESCUELA PROVINCIAL DE PUERICULTURA DE GIJÓN}

La Escuela Provincial de Puericultura de Gijón data de 1927 y es la primera Escuela de Puericultura de España, después de la Nacional . Estuvo emplazada desde su fundación, en la Plaza de Los Mártires $n^{\circ} 1$, en el edificio de la Gota de Leche, donde actualmente se conserva su aula y biblioteca, de enorme belleza por su decoración en madera y cerámica de Talavera. Esta Escuela formaba parte del Instituto de Puericultura de la Junta de Protección de Menores de Gijón y fue establecida amparándose en la disposición del Decreto de 1925, por el que se creaba la Escuela Nacional de Puericultura y según el cual, podian establecerse escuelas provinciales y locales. Se autoriza el funcionamiento de dicha escuela en la que se han de "cursar los estudios elementales que se indican en relación a los importantes problemas que afectan a la salud de la madre y el niño"; y se celebra la inauguración de la primera escuela provincial dentro del territorio español, el dia 30 de Octubre de 1927.

Aunque las enseñanzas teóricas en la formación de enfermeras, fueron en ocasiones, relativamente inconexas y alejadas de la realidad y sobre todo de las necesidades de la población, tal situación no puede hacerse extensiva a las enseñanzas de las Escuelas de puericultura cuya misión estaba enfocada a la instrucción de madres y de todo tipo de profesionales sanitarios y docentes, estimulando la investigación en el terreno de la Puericultura.

Lo más destacable de la Escuela de Puericultura gijonesa es que logra ser la primera escuela de España, después de la Nacional, obteniendo reconocimiento nacional e internacional por sus enseñanzas, recursos y modernos equipamientos, haciéndola una Institución modélica al estar unida al Instituto de Puericultura de la misma localidad.

En las enseñanzas que se imparten en esta Escuela además de las técnicas sanitarias, se hace hincapié en la Educación Sanitaria, Higiene Alimentación, Epidemiología y Psicología Infantil. Se inculcan, sin hacerlo explícito, las teorías de Florence Nightingale, con los conceptos principales de ventilación, calor, dieta, limpieza, ruido y todos los componentes medio - ambientales Muestra de ello, son los carteles divulgativos que utilizan para la Educación Sanitaria de las madres .

Los profesores son todos médicos y varones, con excepción de la Enfermera encargada de las alumnas. En un principio la voluntariedad de los mismos hizo que se impartieran más horas y asignaturas de las debidas, todo elllo con sólo un objetivo: "crear un cuerpo de Enfermeras que honren a la Escuela y a Gijón".

Los cursos que convoca la Escuela son para personal femenino exclusivamente (Enfermeras, maestras y matronas puericultoras, Ayas o cuidadoras de niños y auxiliares de puericultura) con excepción de los convocados para el título de médicos puericultores o maternólogos.

Podemos establecer tres períodos dentro de la existencia de dicha Escuela:

- Inaugural y de desarrollo incipiente, caracterizado por la voluntariedad y entusiasmo de los pione- 
ros, ayudados por el pueblo de Gijón con la Rifa de La Pro-Infancia . En las solicitudes de títulos de las primeras alumnas de la Escuela de Gijón, se especifica su profesión de enfermera y/o matronas y el examen de ingreso en la Escuela, se realizaba ante un Tribunal compuesto por el Jefe Provincial de Sanidad, José $\mathrm{M}^{a}$ Gasset, el Director de la misma Avelino Gonzalez y el médico maternólogo del estado, Alejandro Fournier.

- De esplendor, en el que subvencionada por organismos centrales comienza su actividad más importante, en la disminución de la mortalidad infantil y control de la Tuberculosis. De esta Escuela salen enfermeras y matronas puericultoras, que tras la inauguración del Hospital General, pasarán a formar parte de su plantilla como son Ángeles Fernández Rodríguez, Nieves Romero Vázquez y Aurora González de Bernardi.

En 1935 se reorganiza la Escuela Nacional de Puericultura y se conservarán sólo tres escuelas provinciales las de Sevilla, Valencia, y Bilbao, haciendo dudar de los efecțos oficiales de los estudios cursados en la Escuela de Gijón y poniendo en tela de juicio la oficialidad y continuidad de la misma. Este hecho origina la réplica del Dr. Avelino Glez . en estos términos : "... En ninguna disposición legal se ordena el cese de la misma, ni taxativamente, ni con carácter general, pudiéndose sobrentender que la escuela de Gijón será conservada, pues de no ser así se había prohibido oficialmente su funcionamiento y como no ha sido designada, como una de las tres escuelas Provinciales viene sujeta a la legislación anterior..."

Así se inagura el curso en Octubre de 1939, con la presencia entre otros, del Director General de Sanidad Dr. J. A. Palanca y el Dr. Suñer Director de la Escuela Nacional de Puericultura, decidiendo que dependa esta escuela del servicio de Sanidad Nacional, comenzando a percibir de este organismo, en 1940, y tras 13 años de funcionamiento, la subvención de 2.500 pts. trimestrales, ascendiendo a 5.000 pts. en Enero de 1941

En 1958 se solicita de Madrid, información sobre la conveniencia de potenciar la Escuela o suprimirla, respondiendo a ello, afirmativamente y alegando:

La antigüedad y prestigio de la Escuela.

La necesidad de promocionar personal con preparación puericultora, por ser Gijón y su zona satélite una región en desarrollo con problemas de higiene infantil urgente y específicos.

La vinculación a la Junta Local de Protección de menores, como está previsto en el reglamento de las Escuelas de Puericultura, lo que mejora extraordinariamente la situación presupuestaria.

Al faltar en Gijón otros organismos que permitan la preparación sanitaria del personal femenino. (1960).

De cambios, que transcurren a lo largo de esta etapa tanto a nivel normativo, como institucional y en la que el Dr. Avelino Gonzalez se queja de se está perdiendo el concepto de Puericultura. Los documentos que a continuación expongo, nos dejan entrever la lucha que se mantuvo por la Puericultura, llegando el Dr. Avelino Glez., a diferenciarla de la Pediatría y escribiendo: " En Gijón se ha obtenido un gran descenso de la Mortalidad, pasando de un $17,5 \%$ en 1920 , y tras realizar intensa propaganda con conferencias, artículos, formación etc., se llegó a disminuir al 4,7\% y esto fue debido a la Puericultura y no a la Pediatría... ¿Quién llega a los pueblos remotos, alejados docenas de Kilómetros de las pequeñas poblaciones? Es el personal sanitario y no debemos descuidar este personal que hacemos en nuestras Escuelas modesto y humilde, pero absolutamente interesante... Dada mi larga experiencia de 42 años, en que habiendo estudiado a fondo la Pediatría, vi que no se podía realizar una labor social eficaz y segura de no desviarse a la Puericultura". Los Servicios de Puericultura Centrales solicitan de Don Avelino, 
que les envíe fotos y un breve resumen del Instituto de Puericultura de Gijón y de su escuela con motivo de la Exposición Internacional de Caridad en Roma y en el apartado de historia de la Institución se lee: "...Está considerada la ciudad de Gijón, como la ciudad más importante de Puericultura de España." Es en 1964, cuando Don Avelino se jubila a los 70 años de edad, y es nombrado director Don Pedro Víctor Álvarez, y con el transcurso del tiempo y tras la aparición de las escuelas de A.T.S. en Asturias, se dedican a la formación de auxiliares de puericultura (1969), se cerrando definitivamente sus puertas a la docencia en 1984.

Por último, quiero reseñar, que tras los datos expuestos podemos decir que la Escuela Provincial de Puericultura de Gijón, marcó un hito importante en la Enfermería Materno - infantil, en Asturias por:

- Ser la primera en su categoría (provincial), en España.
- El cambio tan significativo en la orientación de los cuidados enfermeros, haciéndolos pasar de un carácter eminentemente técnico a otro reparador y de mantenimiento de la vida.

- La formación de personal multidisciplinario, altamente preparado para los cuidados a la madre y al niño, y que colaboró en la disminución de la mortalidad infantil especialmente en Gijón.

- La utilización de técnicas de Educación Sanitaria y la difusión de la necesidad de cuidados maternoinfantiles para la salud de la población

Ya pongo punto final de este trabajo con una cita, de Fernandez de Pinedo que tiene sus orígenes en el movimiento obrero y dice:

"La salud no se vende, se defiende. No se puede defender lo que no se ama y no se puede amar lo que no se conoce".

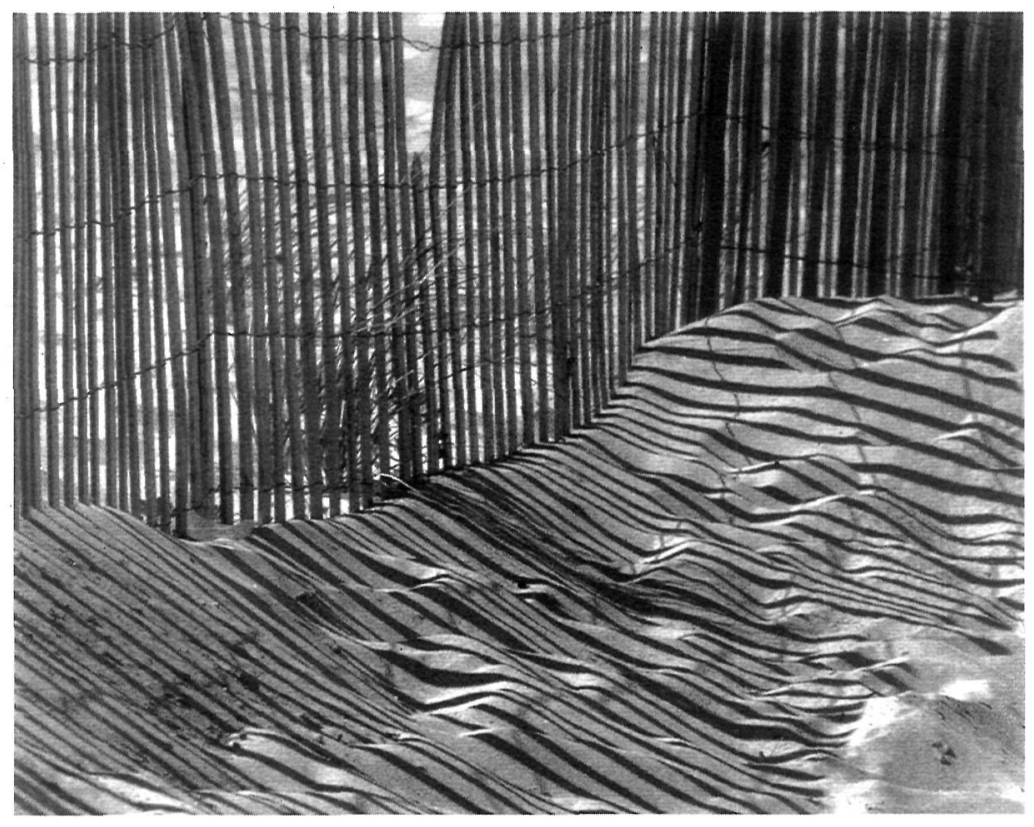

